Correspondencia a:

${ }^{1}$ Laboratorio Clínico 16 de Julio.

La Paz - Bolivia.

Procedencia y arbitraje:

No comisionado, sometido a arbitraje externo

Recibido para publicación: 18 de agosto del 2021

Aceptado para publicación: 28 de octubre del 2021

Citar como:

Alarcón (†) L, Terán Ventura E. Enteroparasitosis en escolares de Huaricana y Viacha. Recisa UNITEPC. 2021; 8(2): 44-52.

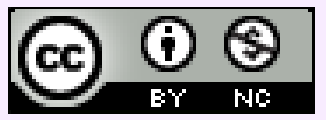

Esta obra está bajo una Licencia Creative Commons Atribución-NoComercial 4.0 Internacional.

\section{Enteroparasitosis en escolares de Huaricana y Viacha d}

\section{Enteroparasitosis in schoolchildren from Huaricana and Viacha}

\section{Enteroparasitose em escolares de Huaricana e Viacha}

Luis Alarcón $\dagger^{1}$ PhD. Evangelina Terán Ventura ${ }^{1}$

\section{Resumen}

Introducción: La parasitosis intestinal constituye una de las infecciones más comunes a nivel mundial, de mayor prevalencia en países poco desarrollados. El objetivo es comparar la prevalencia de enteroparásitos en niños de Huaricana y Viacha de La Paz, Bolivia. Metodología: Se colectaron muestras de heces de niños de $4-11$ años de los colegios de Huaricana $(H)$ y Viacha (V). Para determinar la presencia de enteroparásitos, se utilizó la técnica modificada de concentración de Willis y Ritchie. Resultados: La prevalencia de enteroparásitos fue de más del $80 \%$ para $\mathrm{H}$ y $44 \%$ para $\mathrm{V}$. Las especies de helmintos que fueron encontrados son: Hymenolepis nana $(\mathrm{H}: 20 \%, \mathrm{~V}$ : $0 \%)$, Ascaris lumbricoides ( $\mathrm{H}: 4 \%, \mathrm{~V}: 4 \%)$, Enterobius vermicularis $(\mathrm{H}: 5 \%$, V: $0 \%)$, Taenia spp. (H: $4 \%$, V: $0 \%)$, Diphylidium spp. (H:4\%, $: 0 \%$ ), y entre los protozoos se encontraron Giardia lamblia ( $\mathrm{H}: 18 \%, \mathrm{~V}: 4 \%)$, Blastocystis hominis $(\mathrm{H}: 22 \%$, $\mathrm{V}: 6 \%)$, Entamoeba coli (H:51\%, V:28 \%), Chilomastix mensnili (H:12\%, V:2 \%), Endolimax nana (H:0 \%, V:6 \%) y lodamoeba butschili (H: $14 \%$, V: $2 \%$ ). Los enteroparásitos patógenos predominantes en Huaricana fueron Hymenolepis nana $20 \%$ y Giardia lamblia $18 \%$; y en Viacha $4 \%$ se encontraron Ascaris lumbricoides y Giardia lamblia. Los niños de Huaricana estaban poliparasitados en $20 \%$, y biparasitados en $25 \%$, y los niños de Viacha estaban biparasitados en $19 \%$. Conclusión: Los niños con mayor prevalencia de enteroparásitos pertenecen a Huaricana. Las especies patógenas de enteroparásitos prevalentes en niños de Huaricana fue Hymenolepis nana, Giardia lamblia y de Viacha fue Ascaris lumbricoides y Giardia lamblia. Los niños de Huaricana estaban poliparasitados y los de Viacha estaban biparasitados.

Palabras Clave: Parasitosis Intestinales, Estudiantes, Prevalencia.

\section{Summary}

Introduction: Intestinal parasitosis is one of the most common 
infections worldwide, with the highest prevalence in less developed countries. The objective is to compare the prevalence of enteroparasites in children from Huaricana and Viacha de La Paz, Bolivia. Methodology: Stool samples were collected from children aged 4-11 years from the schools of Huaricana $(\mathrm{H})$ and Viacha $(\mathrm{V})$. To determine the presence of enteroparasites, the modified Willis and Ritchie concentration technique was used. Results: The prevalence of enteroparasites was more than $80 \%$ for $\mathrm{H}$ and $44 \%$ for $\mathrm{V}$. The helminth species that were found are: Hymenolepis nana $(\mathrm{H}: 20 \%, \mathrm{~V}$ : $0 \%)$, Ascaris lumbricoides $(\mathrm{H}: 4 \%, \mathrm{~V}: 4 \%)$, Enterobius vermicularis $(\mathrm{H}: 5 \%, \mathrm{~V}: 0 \%)$, Taenia spp. (H: 4\%, V: 0\%), Diphylidium spp. $(\mathrm{H}: 4 \%, \mathrm{~V}: 0 \%)$, and among the protozoa were Giardia lamblia (H: 18\%, V: 4\%), Blastocystis hominis (H: 22\%, V: 6\%), Entamoeba coli $(\mathrm{H}: 51 \%$, V: $28 \%)$, Chilomastix mensnili $(\mathrm{H}: 12 \%, \mathrm{~V}: 2 \%)$, Endolimax nana $(\mathrm{H}$ : $0 \%, \mathrm{~V}: 6 \%$ ) and lodamoeba butschili ( $\mathrm{H}: 14 \%, \mathrm{~V}: 2 \%)$. The predominant pathogenic enteroparasites in Huaricana were Hymenolepis nana $20 \%$ and Giardia lamblia $18 \%$; and $4 \%$ of Viacha found Ascaris lumbricoides and Giardia lamblia. The Huaricana children were polyparasitized in $20 \%$, and biparasitized in $25 \%$, and the Viacha children were biparasitized in 19\%. Conclusion: The children with the highest prevalence of enteroparasites belong to Huaricana. The pathogenic species of enteroparasites prevalent in children from Huaricana was Hymenolepis nana, Giardia lamblia and from Viacha it was Ascaris lumbricoides and Giardia lamblia. The children of Huaricana were polyparasitized and those of Viacha were biparasitized.

Keywords: Intestinal Diseases Parasitic, Students, Prevalence.

\section{Resumo}

Introdução: A parasitose intestinal é uma das infecções mais comuns em todo o mundo, com maior prevalência em países menos desenvolvidos. O objetivo é comparar a prevalência de enteroparasitas em crianças de Huaricana e Viacha de La Paz, Bolívia. Metodologia: Amostras de fezes foram coletadas de crianças de 4 a 11 anos das escolas de Huaricana $(\mathrm{H})$ e Viacha $(V)$. Para determinar a presença de enteroparasitas, foi utilizada a técnica de concentração modificada de Willis e Ritchie. Resultados: A prevalência de enteroparasitas foi superior a $80 \%$ para $\mathrm{H}$ e $44 \%$ para V. As espécies de helmintos encontradas são: Hymenolepis nana $(\mathrm{H}: 20 \%, \mathrm{~V}: 0 \%)$, Ascaris lumbricoides $(\mathrm{H}: 4 \%, \mathrm{~V}: 4 \%)$, Enterobius vermicularis $(\mathrm{H}: 5 \%, \mathrm{~V}: 0 \%)$, Taenia spp. (H: 4\%, V: 0\%), Diphylidium spp. (H: 4\%, V: 0\%), e entre os protozoários estavam Giardia lamblia (H: 18\%, V: 4\%), Blastocystis hominis (H: 22\%, V: 6\%), Entamoeba coli $(\mathrm{H}: 51 \%$, V: $28 \%)$, Chilomastix mensnili $(\mathrm{H}: 12 \%, \mathrm{~V}: 2 \%)$, Endolimax nana $(\mathrm{H}: 0 \%, \mathrm{~V}$ : $6 \%)$ e lodamoeba butschili $(\mathrm{H}: 14 \%$, V: $2 \%)$. Os enteroparasitas patogênicos predominantes em Huaricana foram Hymenolepis nana 20\% e Giardia lamblia 18\%; e 4\% da Viacha encontrou Ascaris lumbricoides e Giardia lamblia. As crianças Huaricana eram poliparasitárias em 20\%, e biparasitadas em 25\%, e as crianças Viacha eram biparasitadas em 19\%. Conclusão: As crianças com maior prevalência de enteroparasitas pertencem a Huaricana. As espécies patogênicas de enteroparasitas prevalentes em crianças de Huaricana foram Hymenolepis nana, Giardia lamblia e de Viacha foram Ascaris lumbricoides e Giardia lamblia. Os filhos de Huaricana foram poliparasitados e os de Viacha foram biparasitados.

Palavras chaves: Enteropatias Parasitárias, Estudantes, Prevalência. 


\section{Introducción}

Hoy en día las enfermedades parasitarias constituyen un problema de Salud Pública, por su elevada frecuencia en países en vías de desarrollo, y su incidencia en países desarrollados, se debe a la migración de individuos provenientes de zonas del tercer mundo y por su alta morbilidad (1).

La Organización Mundial de la Salud estima que más de 2 billones de personas en el mundo viven con enfermedades debido a los parásitos intestinales especialmente en países en vías de desarrollo siendo la mayoría niños (2).

La OPS/OMS calcula que una de cada tres personas está infectada por geohelmintos y cerca 46 millones de niños entre 1 y 14 años están en riesgo de infectarse por estos parásitos, aproximadamente $28 \%$ en edad preescolar y $72 \%$ en escolares. Por falta de saneamiento básico y acceso de agua potable, son las infecciones más comunes a nivel mundial y afectan a las poblaciones pobres y vulnerables, mujeres e infantes (3).

Estas afecciones son conocidas como enfermedades desatendidas, por la poca importancia que dan los gobiernos y por ser consideradas de baja prioridad de salud pública internacional (4). Las elevadas tasas de infestación por parásitos intestinales en países latinoamericanos son un reflejo de la situación en la que viven sus habitantes, en ocasiones persistentemente expuestos a un entorno contaminado con microorganismos patógenos, además de las deficiencias en los hábitos de higiene (5).

Frecuentemente, la elevada prevalencia de enteroparasitosis, está relacionada con la contaminación fecal del agua de consumo y suelo, o de los alimentos añadidos los deficientes condiciones sanitarias, de educación, higiene y socioculturales $(6,7)$ Argentina were analysed. Coproparasitological screenings were performed in children up to 14 years old from a marginal zone (100.

La información sobre incidencia y prevalencia de enteroparasitos en Bolivia, es insuficiente y con deficiencias técnicas en su procesamiento en las fases: pre-analítica, analítica y post-analítica, de trabajos de investigación, que no siempre aplican las reglas de estudio, procedimientos de laboratorio inadecuados, y tampoco proporcionan datos epidemiológicos confiables (8).

Por tanto, nuestro objetivo fue comparar la prevalencia de enteroparasitosis en niños de 2 colegios de diferentes zonas, "El Rosario" de Huaricana y "Gualberto Villarroel" de Viacha, de La Paz - Bolivia.

\section{Metodología}

Mecapaca es un municipio de Bolivia, ubicado en la provincia Murillo del departamento de La Paz. Se encuentra a $28 \mathrm{~km}$ de la ciudad, y se localiza a 2.850 metros sobre el nivel del mar. Según el censo nacional de 2012, tiene una población de 16.456 habitantes mayores de 18 años (9).

Mecapaca es la segunda sección municipal de la provincia Murillo. Al norte limita con el municipio de La Paz, al este con el de Palca al sur con las provincias Aroma y Loayza, al oeste con Achocalla. Su topografía es montañosa, con quebradas pronunciadas que se precipitan sobre el río de La Paz, lo cual atraviesa todo su territorio. El clima mantiene diferencias estacionales marcadas, con una temperatura promedio de $18^{\circ} \mathrm{C}$. Más del $40 \%$ de los hogares dispone de energía eléctrica. Los servicios de 
salud son muy precarios con siete centros de atención primaria (10). Parte de la población de Huaricana reciben agua de lluvia o acequia, y el desagüe de servicio sanitario tiene pozo ciego y en la quebrada (9).

Villa de Viacha o Viacha es un municipio de Bolivia, además de ser la capital de la provincia de Ingavi ubicada en el departamento de La Paz. Su población es de 80724 habitantes. Está a $22 \mathrm{~km}$ de la ciudad de La Paz. Se encuentra en el Altiplano boliviano y posee un clima frío y seco la mayor parte del año con una estación lluviosa entre diciembre y febrero. La mayoría cuenta con agua de cañería de red, y el desagüe de servicio sanitario tiene alcantarillado y pozo ciego (9).

La población de estudio fueron niños de 5-12 años de edad, de 2 colegios "El Rosario" de Huaricana $(\mathrm{H}) \mathrm{n}=74$ y "Gualberto Villarroel" de Viacha $(V) n=47$, ambos del departamento de La Paz.

Se recolectaron muestras de heces en frascos plásticos desechables con tapa rosca (tres por estudiante), en la respectiva institución. A cada participante se le prescribió un análisis coproparasitologico seriado emitido espontáneamente. Estas se procesaron mediante la técnica directa y la de concentración modificada Ritchie (1).

Después de observar al microscopio por la técnica directa, se añadió formol al $5 \%$ para conservar la muestra. Luego se procedió con la de concentración modificada Ritchie en el laboratorio 16 de julio de la ciudad de La Paz, agregando solución salina, se filtró por una capa doble de gasa, y se centrifugó a 1000 gravedades durante 3 minutos.

Tanto con la técnica directa y la de concentración modificada se observaron al microscopio (Olympus) trofozoitos y quistes de protozoos, así como de huevos y larvas de helmintos, con aumentos de 10X, y 40X, con una gota de lugol y otra de solución salina, con 2 observadores diferentes para cada estudio.

Previo a la recolección de las muestras, se realizó un plan de acción que incluyó actividades de promoción y prevención sobre parasitosis intestinales. Todos los adultos y los responsables de los menores participantes firmaron una declaración de "Consentimiento Informado", en el que quedó escrito el deseo de colaborar voluntariamente. Este protocolo fue aprobado por el comité nacional de ética de investigación científica de la Universidad Mayor de San Andrés de la ciudad de La Paz - Bolivia.

Se calcularon las frecuencias totales de prevalencia de parasitosis, y tipo de parásitos, para el ensayo se utilizaron las pruebas de chi-cuadrado. Los análisis estadísticos se realizaron con el programa informático GraphPad Prism versión 4.1. Se consideraron valores significativos con una significación de $P<0,05$.

\section{Resultados}

La población de estudio fueron niños de 5 a 12 años de edad, de 2 colegios "El Rosario" de Huaricana $(H) n=74$ y "Gualberto Villarroel" de Viacha $(V) n=47$, ambos del departamento de La Paz.

Nuestros resultados reportan diferencias estadísticamente significativas de la prevalencia de enteroparasitosis en los niños de ambos colegios: en el Rosario de Huaricana de 74 estudiantes 64 estaban parasitados haciendo un $86 \%$ y en Gualberto Villarroel de Viacha de 47 infantes 21 con un $44 \%$ para V, (Chi-square; $P=0,0001$ ). 
Los niños de Huaricana y Viacha estaban poliparasitados, biparasitados y monoparasitados como muestra la Tabla 1 (Chi-square; $P=0,04$ ).

Tabla 1. Prevalencia (\%) de niños de los Colegios "El Rosario" de Huaricana y el Colegio "Gualberto Villarroel" de Viacha, mono, bi y poliparasitados; chi-cuadrado $\mathrm{P}=0,04$ *.

\begin{tabular}{|l|l|l|l|l|}
\hline Número de parásitos & $\begin{array}{l}\text { Colegio "El Rosario" } \\
\text { Huaricana }\end{array}$ & $\%$ & $\begin{array}{l}\text { Colegio "Gualberto } \\
\text { Villarroel" Viacha }\end{array}$ & $\%$ \\
\hline Monoparasitados & 36 & 55 & 17 & 81 \\
\hline Biparasitados & 16 & 25 & 4 & 19 \\
\hline Poliparasitados & 13 & 20 & 0 & 0 \\
\hline Total & 65 & 100 & 21 & 100
\end{tabular}

De acuerdo al análisis estadístico chi-cuadrado de los tipos de parásitos, se encontraron estadísticamente diferencias entre las poblaciones estudiadas (Chi-square; $P=0,0001)$, se hallaron helmintos, tales como Ascaris lumbricoides, también se encontró Dipylidium sp., Hymenolepis nana, Taenia spp., Enterobius vermicularis, y entre los protozoos se encontraron Giardia lamblia, Entamoeba coli, Iodamoeba butschlii, Blastocystis hominis y Chilomastix mesnili, como muestra la Figura 1

Figura 1. Prevalencia por tipo de protozoos y helmintos (\%) en el Colegio "El Rosario" de Huaricana y en el colegio "Gualberto Villarroel" de Viacha. La Paz-Bolivia; Chi-square; $P=0,0001$.

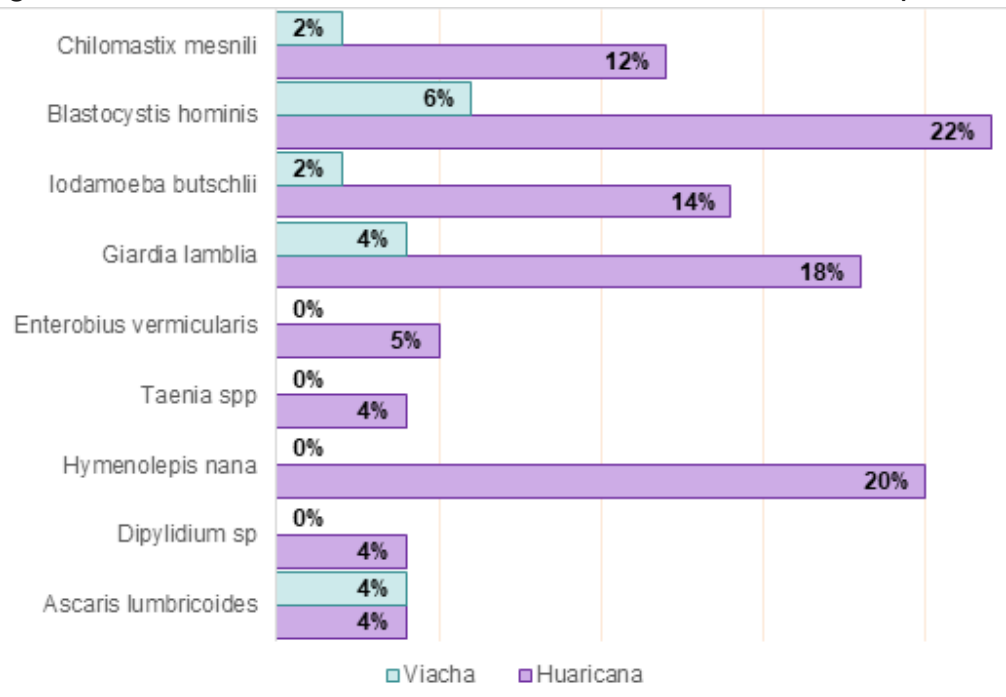

Los enteroparasitos patógenos helmintos y protozoos que predominaron en los niños de Huaricana fueron Hymenolepis nana con $20 \%$ y Giardia lamblia con $18 \%$; y en los niños de Viacha con un $4 \%$ se encontraron Ascaris lumbricoides y Giardia lamblia.

En las dos poblaciones de niños encontramos mayor cantidad de protozoos con $75 \%$ para los niños del Colegio de Huaricana y $92 \%$ para los niños del Colegio Viacha (Tabla 2).

Tabla 2. Prevalencia porcentaje de helmintos y protozoos en niños del Colegio "El Rosario" de Huaricana y en el colegio "Gualberto Villarroel" de Viacha. La Paz-Bolivia; chi-cuadrado $\mathrm{P}=0,0001$

\begin{tabular}{l|l|l|l|l|} 
Enteroparasito & $\begin{array}{l}\text { Colegio "El Rosario" } \\
\text { Huaricana }\end{array}$ & $\begin{array}{l}\text { Colegio "Gualberto } \\
\text { Villarroel" Viacha }\end{array}$ & $\%$ \\
\hline Helmintos & 28 & 25 & 2 & 8 \\
\hline Protozoos & 86 & 75 & 23 & 92 \\
\hline Total & 114 & 100 & 25 & 100 \\
\hline
\end{tabular}




\section{Discusión}

En el presente estudio, se puede observar de manera evidente que la tasa de prevalencia de enteroparasitosis en niños en edad escolar es más alta en el colegio de Huaricana que en el de Viacha. Probablemente sea porque en este ultimo la mayor parte de su población recibe agua de cañería de red, y el desagüe de servicio sanitario tiene alcantarillado y pozo ciego (9).

En cambio, parte de la población de Huaricana reciben agua de lluvia o acequia, y el desagüe de servicio sanitario tiene pozo ciego y en la quebrada (10).

La elevada tasa de enteroparasitosis más del $80 \%$ en Huaricana es similar a otras provincias de Bolivia y también de países latinoamericanos y otros, donde se han encontrado todavía alta prevalencia de parasitosis intestinales. Así en río Abajo, La Paz (71\%), Quillacollo, Cochabamba (97,22 \%), Ixiamas (90\%) (13), Orinoco, Venezuela $85.59 \%$, estado de Mato Grosso do Sul, Brasil (76,9 \%), North Sumatra, Indonesia $(57,24 \%)(11-15)$.

En este estudio el $20 \%$ de los infantes del Colegio de Huaricana estaban poliparasitados, observándose en algunos más de dos especies de parásitos, al contrario, en el de Viacha no se observaron niños con esta infección multiparasitaria, el 19 \% estaba biparasitados.

El hallazgo de Huaricana difiere a los resultados preliminares obtenidos por Aguilar que indican un $67 \%$ de poliparasitismo en el sector de Yupampa lugar cercano, perteneciente al mismo municipio. Hecho que podría deberse al tiempo transcurrido más de 10 años, desde el estudio hasta la fecha, donde las postas se han convertido en centros de salud de atención primaria, y han aumentado las desparasitaciones masivas en los niños.

Similares resultados de poliparasitismo fueron reportados por Cando-Brito en una población infantil del distrito Riobamba, Chambo de Ecuador con un $17 \%$. Superiores se reportan en Quillacollo, Cochabamba, Bolivia y Unión Juárez, Chiapas, México donde fue de $61,11 \%$ y $28 \%$, respectivamente $(16,17)$.

Huaricana área rural de La Paz, Boliva, es un lugar con un clima templado, húmedo apto para la sobrevivencia de ratas; donde las malas condiciones higiénicas, la falta de agua potable y alcantarillado, se hacen propicias para la transmisión de $\mathrm{H}$. nana a los humanos. Aunque este colegio tiene inodoros y pilas para lavarse las manos, no hay suficiente líquido elemento para su uso, por tanto; los niños están expuestos a esta parasitosis.

Nuestros datos de Huaricana muestran predominancia de Hymenolepis nana, el hallazgo coincide con resultados preliminares de Aguilar, donde se encuentra la parasitosis en mayor cantidad con respecto a los helmintos, en Yupampa, rio abajo cerca de esta población.

Es así que, en Cochabamba, Bolivia, de clima similar a Huaricana, también encontraron a Hymenolepis nana como el helminto más frecuente (18). Igualmente, en otros países, se determinó una mayor infección por este parásito en una comunidad indígena del estado Bolívar, Venezuela (19), y en el Sur de España (20).

Los datos de Viacha muestran que el helminto con mayor frecuencia fue A. lumbricoi- 
des, dato que coincide con Peréz G. en Santiago de Cuba, donde uno de los helmintos constante fue este parásito. Un estudio en Puno, lugar de similares características climáticas que esta localidad, indica todo lo contrario (21).

Los protozoarios tienen un comportamiento peculiar porque su prevalencia mayor o menor es dependiente de factores condicionantes de estas infecciones (higiene personal precaria, carencia de servicios básicos, pobreza, entre otros aspectos) (8), (26). Nuestros resultados muestran elevada frecuencia de protozoos con relación a helmintos, en ambos colegios, así como reporta Cando Brito $(16,22)$.

El tipo de protozoo con mayor frecuencia en los niños de ambos colegios fue Entoameba coli, seguido de Blastocystis hominis. Así reportan en Ecuador las mismas características encontradas. Este parásito es considerada no patógeno, relacionada con la obesidad en infantes portadores como indica Zavala (23).

El protozoo B. hominis causa eliminación de sangre por heces fecales, lo cual podría ocasionar anemia como indica Javaherizadeh (24). Es así que en nuestro estudio el predominio de esta parasitosis en Huaricana probablemente sea consecuencia del inadecuado saneamiento y abastecimiento de agua (26), aunque se mencionó anteriormente el Colegio tiene inodoros y pilas para lavarse las manos, pero no hay suficiente líquido elemento para su uso.

Nuestros datos muestran alta frecuencia de Giardia lamblia en niños de Huaricana, probablemente este protozoo sea la causa de diarreas en esta población, así como menciona Díaz G. que la diarrea y parasitosis es una de las afecciones que aquejan a infantes de Mecapaca, distrito al que pertenece.

En conclusión, los resultados evidencian que los niños con mayor prevalencia de enteroparasitos pertenecen a la zona de Huaricana. La especie de parásito patógeno encontrada fue; Hymenolepis nana y Giardia lamblia, de Viacha fue Ascaris lumbricoides, y el mismo protozoario.

Los niños de la zona Huaricana estaban poliparasitados a diferencia de Viacha se encontraban biparasitados.

\section{Conflicto de intereses}

Ninguno

\section{Referencias bibliográficas}

1. Botero D, Restrepo M. Parasitosis Humanas. Incluye animales venenosos y ponzoñosos. 5ta ed. Medellin Colombia: Corporacion para Investigaciones Biológicas; 2012.

2. Desparasitación para la salud y el desarrollo: informe de la tercera reunión mundial de socios para el control de parásitos [Internet]. Ginebra, Suiza: World Health Organization; 2005 [citado 11 de febrero de 2021]. Report No.: WHO/CDS/CPE/ PVC/2005.14. Disponible en: https://apps.who.int/iris/handle/10665/69005

3. $\mathrm{PAHO} / \mathrm{WHO}$. Helmintiasis transmitida por el suelo [Internet]. 2017 [citado $11 \mathrm{de}$ febrero de 2021]. Disponible en: https://www.paho.org/en/topics/soil-transmitted-helminthiasis

4. Holveck JC, Ehrenberg JP, Ault SK, Rojas R, Vasquez J, Cerqueira MT, et al. Pre- 
vention, control, and elimination of neglected diseases in the Americas: pathways to integrated, inter-programmatic, inter-sectoral action for health and development. BMC Public Health. 2007;17(1):7-6.

5. lannacone J, Benites MJ, Chirinos L. Prevalencia de infección por parásitos intestinales en escolares de primaria de Santiago de Surco, Lima, Perú. Parasitol Latinoam. 2006;61(1-2):54-62.

6. Gamboa MI, Basualdo JA, Córdoba MA, Pezzani BC, Minvielle MC, Lahitte HB. Distribution of intestinal parasitoses in relation to environmental and sociocultural parameters in La Plata, Argentina. J Helminthol. 2003;77(1):15-20.

7. Sackey M, Weigel MM, Armijos RX. Predictors and Nutritional Consequences of Intestinal Parasitic Infections in Rural Ecuadorian Children. J Trop Pediatr. 2003;49(1):17-23.

8. Mollinedo J. El Enteroparasitismo en Bolivia - memoria de la Investigación 1975 2004. 2006.

9. Instituto Nacional de Estadística [Internet]. INE. [citado 11 de febrero de 2021]. Disponible en: https://www.ine.gob.bo/

10. Municipalida de Mecapaca (Bolivia) [Internet]. Cooperanda. [citado 11 de febrero de 2021]. Disponible en: https://cooperanda.org/explorar/contrapartes/ver/municipalida-de-mecapaca-bolivia/

11. Mamani-Ortiz Y, Rojas-Salazar EG, Choque-Ontiveros M del C, Caero-Suarez $\mathrm{RI}$. Relación entre la escolaridad y la incidencia de enteroparasitosis infantil en la ciudad de Quillacollo. Rev Méd-Científica Luz Vida. 2012;3(1):6.

12. Teran Ventura E, Rizzo S. Incidencia de parasitosis y relación con anemias en niños de comunidades de la provincia Abel Iturralde-Ixiamas. Rev Científica Salud UNITEPC. 2017;2(1):15-31.

13. Gasteaburu PK. Prevalencia de parasitosis intestinales en niños indígenas Warao y criollos de Barrancas del Orinoco. Venezuela. CIMEL. 2019;24(1).

14. Neres-Norberg A, Guerra-Sanches F, Blanco Moreira-Norberg PR, Madeira-Oliveira JT, Santa-Helena AA, Serra-Freire NM. Enteroparasitismo en Indígenas Terena en el Estado de Mato Grosso do Sul, Brasil. Rev Salud Pública. diciembre de 2014;16(6):859-70.

15. Pasaribu AP, Alam A, Sembiring K, Pasaribu S, Setiabudi D. Prevalence and risk factors of soil-transmitted helminthiasis among school children living in an agricultural area of North Sumatera, Indonesia. BMC Public Health. 2019;19(1):1066.

16. Brito VMC, Arrieta SNE, Chávez CEE, Sánchez ERC. Prevalencia De Parasitosis Intestinales Y Su Posible Relaciòn Con Estados Anèmicos En Los Niños Que Acuden A Los Centros De Educaciòn Inicial. Eur Sci J ESJ. 30 de septiembre de 2017;13(27):113-113.

17. Arana MÁM, Gaspar M del RA, Aguilar FJR, Vizuet MGT. Prevalencia de parasitosis intestinal en niños de guarderías rurales en Chiapas. Enfermedades Infecc Microbiol. 2020;40(2):43-6.

18. Zurita Céspedes BI, Moya Álvarez RR, Moya Álvarez KL, Tellez León TM, Torrico 
Rojas MC. Frecuencia de parásitos intestinales en exámenes coproparasitológicos directos procesados en el laboratorio de investigación médica, 2011-2015. Rev Científica Cienc Médica. 2018;21(2):6-12.

19. Devera RA, Blanco YY, Rivas N del VV, Rodríguez IDA, Certad I del CR, Nastasi-Miranda J, et al. Infección por Hymenolepis nana en una comunidad indígena del estado Bolívar, Venezuela. Rev Cubana Med Trop. 2016;68(1):70-81.

20. Cabeza MI, Cabezas MT, Cobo F, Salas J, Vázquez J. Hymenolepis nana: factores asociados a este parasitismo en un área de salud del Sur de España. Rev Chil Infectol. 2015;32(5):593-5.

21. Pérez Sánchez G, Redondo de la Fe G, Fong Rodríguez HG, Sacerio Cruz M, onzález Beltrán O. Prevalencia de parasitismo intestinal en escolares de 6-11 años. MediSan. 2012;16(04):551-7.

22. Luna S, Jimenez S, Lopez R, Soto M. Prevalencia de Parasitismo Intestinal en Niños y Mujeres de Comunidades Indígenas del Río Beni. Visión Científica. 2007;1(2):37-34.

23. Zavala GA, García OP, Campos-Ponce M, Ronquillo D, Caamaño MC, Doak $\mathrm{CM}$, et al. Children with moderate-high infection with Entamoeba coli have higher percentage of body and abdominal fat than non-infected children. Pediatr Obes. 2016;11(6):443-9.

24. Javaherizadeh H, Khademvatan S, Soltani S, Torabizadeh M, Yousefi E. Distribution of haematological indices among subjects with Blastocystis hominis infection compared to controls. Przeglad Gastroenterol. 2014;9(1):38-42.

25. Amato Neto V, Rodríguez Alarcón RS, Gakiya E, Bezerra RC, Ferreira CS, Braz LMA. Blastocystosis: controversy and indefinedness. Rev Soc Bras Med Trop. 2003;36(4):515-7.

26. Bastidas G, Rojas C, Martínez-Silva E, Loaiza L, Guzmán M, Hernández V, et al. Prevalencia de parásitos intestinales en manipuladores de alimentos en una comunidad rural de Cojedes, Venezuela. Acta Médica Costarric. 2012;54(4):241-5. 